\title{
Sobre la autoría del Palacio de los Valdés en Gijón: de Juan de Cerecedo, el viejo, a Juan Bautista Portigiani
}

Laura Sampedro Redondo

RESUMEN. Juan de Valdés, regidor de Gijón, acude en 1564 al escribano Juan de Llanos Cifuentes para modificar el contrato que tiene otorgado con Juan Pérez Helguera, cantero, para las obras de las casas que se está haciendo en la villa. Por este contrato, acuerdan dejar de seguir las trazas de Juan de Cerecedo, el viejo, maestro de obras de la Catearal de Oviedo, para seguir desde ese momento una nueva traza realizada por el arquitecto italiano Juan Bautista Portigiani de cuyos detalles se da cuenta en un documento adjunto.

Palabras clave: Juan de Valdés, regidor de Gijón; Juan de Cerecedo, el viejo; Juan Bautista Portigiani, arquitecto.

ABSTRACT. Juan de Valdés, councilman of the town of Gijón, presents himself before the notary Juan de Llanos Cifuentes, with Juan Pérez Helguera, quarryman in charge of building the houses he is making in the town, in order to make some changes in the terms they both had agreed before another notary time ago. The new agreement settles that Juan Pérez Helguera will not follow the design of the Oviedos's Cathedral architect Juan de Cerecedo, the old, any more, and instead, he will follow, from then on, the one given by the Italian architect Juan Bautista Portigiani, whose details they specify in a document attached.

Key Words: Juan de Valdés, councilman of Gijón, Juan de Cerecedo, the old, Juan Bautista Portigiani, Italian architect.

En 1993, Germán Ramallo, en su estudio ${ }^{1}$ sobre "El palacio urbano en Asturias" dedica amplio espacio a comentar los aspectos conocidos acerca de la construcción del Palacio de Valdés en Gijón que define como "un hito para toda la nobleza asturiana que pronto querría imitarlo y superarlo en todo lo que pudiera". Al tiempo que pondera la calidad de la obra, define su estilo como "un sentido clasicista heredado de la fase artística anterior y con un buen conocimiento de los

${ }^{1}$ G. RAMALlO ASENSIO, (Coord). «El palacio urbano en Asturias», Arquitectura señorial en el norte de España, Oviedo, 1993. pp.86 a 90. tratados del Alto Renacimiento" considerando que "tras esta obra tan cuidada se ha de buscar un tracista capaz y al día" pero lamentándose por la falta de datos fehacientes que permitieran poder asignar un nombre a tal proyecto: "cualquier atribución que se haga no dejará de ser pura hipótesis", y por no poder aproximar una fecha de comienzo, más allá del dato seguro que proporciona el "propio dibujo que el mismo Fernando de Valdés mandó hacer de Gijón y sus costas" y que data de 1635. Germán Ramallo da por sentado que tuvo que ser bastante antes, pero la prudencia, ante la falta de datos, no le per- 
mite llegar más allá de situarlo a principios del XVII.

En 1996, Pilar García Cuetos, en su trabajo sobre la arquitectura en Asturias en el siglo $\mathrm{XVI}^{2}$, y más concretamente en las obras atribuidas a Juan de Cerecedo, el viejo, maestro de obras de la Catedral de Oviedo, aporta sin duda valiosos datos que permiten avanzar más allá de la "pura hipótesis" lamentada por Ramallo. Así, el contenido de varias escrituras ${ }^{3}$ : un contrato en el que Cerecedo aparece mencionado como fiador en un contrato anterior de la obra del palacio; un poder entre los canteros para pedir tasación y poder cobrar lo realizado en el palacio a la muerte de Juan Valdés en 1571; y el testamento de uno de los canteros, le permiten calibrar que Juan de Cerecedo, el viejo, podía haber intervenido como maestro tracista -habida cuenta de la categoría profesional de segunda fila de quienes intervienen como canteros en la obra que los sitúa lejos de poder ser uno de ellos el maestro de la misma, y teniendo en cuenta la relevancia social de la persona de Juan de Valdés, y la categoría arquitectónica del edificio construido-. Así García Cuetos acertada y prudente afirma: "probablemente intervino como tracista, Juan de Cerecedo el Viejo" y situa los comienzos de la obra en "los años sesenta del siglo XVI" al entender que "lo más probable es que la fachada y la capilla se añadieran a una estructura que ya estaba cuajada en la segunda mitad del quinientos, incluso es probable que la fachada primitiva ya se hubiera concebido con un cuerpo central y

\footnotetext{
${ }^{2}$ Ma P. GARcía Cuetos, Arquitectura en Asturias 1500-1580, La dinastía de los Cerecedo, Oviedo 1996.

3 , Ma P, GARCÍA CUETOS, Ob.cit., pp. 94-95, la autora resume las partes de tales escrituras que hacen referencia a los hechos, y aporta en notas al pié las signaturas correspondientes. Nota 167, AHP Protocolos de Oviedo, legajo 41, escribano, Alonso Pérez, enero 1570. Nota 168, legajo 42 (I) escribano Alonso Pérez. Y nota 169, legajo 7 del escribano Diego González de Candamo, Testamento y codicilo de Juan de la Roza.
}

dos torres...", aunque líneas antes se lamenta como Ramallo de la falta de datos y afirma "sobre el aspecto y disposición de la residencia construida para Juan de Valdés por esos años, no tenemos ninguna referencia."

Una vez más será la documentación la que nos permita a todos dar un nuevo paso para reducir la incertidumbre, y la que dé razón a la intuición de García Cuetos, porque, en el transcurso de una investigación sobre documentación notarial de Gijón para mi Tesis doctoral, he tenido la fortuna de localizar un documento que despeja la mayor parte -no todas, de nuevo lamentablemente- de las dudas arriba mencionadas, y desde luego, resuelve varias de las hipótesis.

En 1564 Juan de Valdés, regidor de Gijón, acude al escribano de número de la dicha villa, Juan de Llanos Cifuentes ${ }^{4}$ para acordar con Juan Pérez de Helguera, cantero, la modificación de un contrato sobre unas "obras y casas" que éste está realizando para él en la villa de Gijón siguiendo una traza de Juan de Cerecedo. La modificación consiste en que Helguera deje de utilizar la traza de Juan de Cerecedo que Juan de Valdés había contratado con él con anterioridad ante otro escribano, y pase a utilizar la de otro arquitecto. Esto nos permite confirmar que, en efecto, tal y como Ramallo Asensio observaba, la traza era de alguien con conocimientos importantes, y por supuesto da la razón a Pilar García Cuetos cuando acertadamente apunta que la traza del palacio la hace Juan de Cerecedo, el viejo, y que el palacio se empieza a construir a mediados del siglo XVI.

Pero la información no se agota en la alusión a lo que se deja de hacer, sino que nos informa de las nuevas intenciones del regidor gijonés. Esa nueva traza, montea y

\footnotetext{
${ }^{4}$ AHP, Protocolos notariales de Gijón. Caja 1752. Legajo 3, p.11r,11v,12r,12v.
} 
dibujos, que Juan de Valdés quiere que se sigan a partir de ese momento, los realiza y entrega "Juan Battista, arquitecto ytaliano", que firma al final del documento adjunto, como "Ca Battista Portigiani, Arquittecto"; y los aspectos que se modifican, afectan, por lo que se puede ver, a la sillería, ventanas, puertas y otros elementos decorativos, según unas características que especifica en un documento adjunto que, afortunadamente, también se conserva.

Juan Bautista Portigiani aparece documentado por González Santos ${ }^{5}$ como un florentino, posiblemente descendiente de una familia de reconocidos escultores, y formado en el "taller familiar, nutriéndose en la fecunda "maniera" florentina de mediados de siglo, siempre a caballo entre el ineludible magisterio de Miguel Angel y los renovados ecos del clasicismo rafaelesco", que llegó a España en 1563, "atraído por el favor de Felipe II" para las obras del Escorial, donde "encontró patronos tan relevantes, influyentes y cultivados como el arzobispo de Sevilla y Gran Inquisidor, el asturiano don Fernando de Valdés".

En su estudio, González Santos, alude a las "escasas noticias del itinerario español de Portigiani" y esboza lo que el denomina "una biografía de urgencia y carácter provisional" en la que la fecha de partida, para Asturias, es un documento de 19 de enero de 1567, por el que don Hernando de Valdés, canónigo de la Catedral de Oviedo, le contrata en nombre de don Fernando de Valdés, su tío, Arzobispo de Sevilla, para construir el primer retablo de la colegiata de Salas, del que solo se conserva un fragmento, un altorrelieve de una virgen de terracota, Nuestra Señora de la Asunción ${ }^{6}$, cuya

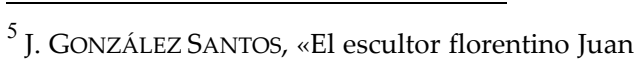
Bautista Portigiani, noticias de sus obras en Asturias» Boletín del Seminario de Estudios de Arte y Arqueología, LII, Valladolid, 1986, pp. 297-310.

${ }^{6}$ J. GONZÁLEZ SANTOS, Los comienzos de la escultura naturalista en Asturias (1575 - 1625) , Oviedo 1997.
}

importancia también recoge Francisco de $\mathrm{Caso}^{7}$ en su estudio sobre la Colegiata de Salas, describiéndola como "una obra de gran interés".

Una vez más, el documento que nos ocupa muestra su enjundia, pues al ser anterior a los mencionados por González Santos y Caso, datados en 1567, sitúa a Portigiani, como arquitecto, en una obra en Gijón en 1564, y eso sí, también de la mano de un Valdés, en esta ocasión el regidor de Gijón, Juan de Valdés.

Toda esta información nos permite observar que, no sólo el palacio empieza a construirse a mediados del XVI, sino que sobre la marcha -hablamos del palacio, de la capilla no se dice nada en absoluto en el documento- se modificó el contrato y se remató la obra iniciada por Cerecedo a la manera del florentino Bautista Portigiani, lo que da la razón a Germán Ramallo cuando observaba que la había planeado alguien "con un buen conocimiento de los tratados del Alto Renacimiento"; al tiempo que gracias a los pormenores descritos, podemos identificar "el aspecto y disposición de la residencia construida para Juan de Valdés por esos años" de la que carecíamos, tal y como apuntaba Pilar García Cuetos.

Por todo lo dicho: nombre del primer tracista, Juan de Cerecedo, nombre del segundo artífice, encargado de darle ese estilo renacentista, el florentino Juan Bautista Portigiani, la fecha que sitúa al arquitecto en Asturias, y que aproxima más la obra, la minuciosa descripción de ventanas, entradas, esquinas y demás que nos aporta el documento de condiciones, creemos que -a falta de otros que esperamos sigan apareciendo producto de investigaciones futuras-, éste es un documento de especial interés para la historia de Gijón, y para la de la

\footnotetext{
${ }^{7}$ F. CASO DE, La colegiata de Salas. Gijón, 1993, pp, 21 y 49 y ss.
} 
arquitectura en Asturias, ya que una vez más, gota a gota, vamos llenando las lagunas.

1564, mayo, 3, Gijón.

Juan de Valdés, tiene contratado a Juan Pérez de Helguera para hacerle obra en sus casas, por un contrato que otorgaron ante el escribano Juan Cerecedo, sobre un diseño de otro Juan Cerecedo, el viejo, maestro de obras de la catedral de Oviedo. Por esta escritura, acuerdan cambiar las condiciones para hacer la obra según el diseño de un arquitecto italiano, Juan Bautista Portigiani, que aporta un nuevo proyecto. Testigos: Fernando de Cifuentes, escribano, Fernando García de Villar, el viejo, y Fernando de Cangas. Firman: Juan de Valdés, Juan Pérez de Helguera y el escribano Juan de Llanos.

Acompaña las condiciones acerca de las nuevas directrices para puertas, ventanas, fachada, material de obra, etc. El documento lo firman, Batista Portigiani, arquitecto, Juan Pérez Helguera, y el escribano Juan de Llanos.

AHPO. Protocolos notariales de Gijón. Juan de Llanos. Caja 1752. Legajo 3.

$/ / 11 \mathrm{r}$

Contrato entre el señor Juan de Baldés e Juan de Felguera.

En la villa de Gijón, a tres días del mes de mayo de mill e quinientos e sesenta e quatro años, ante mí, el escribano público e testigos [re]queridos, paresçieron presentes de la una parte el señor Juan de Baldés, veçino [e] regidor de la dicha villa e conçejo, e de la otra Juan Pérez de Felguera, estante al presente en esta dicha villa, e dixeron que, atento que el dicho Juan Pérez estaba obligado a hazer la obra e casas que el dicho señor Juan de Baldés haze e quiere hazer en esta villa, conforme a la traça que para ellas dio Juan de Zerezedo, maeso de la obra de [...]firmada de su nonbre, según abía pasado el contrato, obligaçión y escritura que sobrello abían hecho e otorgado ante Juan de Zerezedo, escribano, la qual dicha obra al presente tenía el dicho Juan Pérez començada a hazer. E agora Juan Batista, arquiteto ytaliano, abía dado oy dicho día otra nueba traza para que, conforme a ella, se hiziesen, traçasen y hedificasen las dichas casas [e el] dicho Juan Pérez las hiziese y hedificase, la qual traza les par[e]çía más útil, conpetente y nezessaria para la dicha obra e casas, que no la quel dicho Zerezedo abía dado. Por ende, que heran conformes e conçertados en que afirmándose como ante [todas] cosas se afirmaron, cada uno dellos por lo que le toca, en el contrato que entre ellos abía pasado ante el dicho Zerezedo, escribano, y no le alterando ni deshaziendo en cosa ninguna de lo en él contenido, hiziese e pusiese en hefeto en la dicha obra la dicha nueba tra[za] que de presente da e a dado el dicho Juan Batista, con los al[tos] e baxos e dibuxos e moldes, y alto e baxo e largo e asiento de la sillería, e de bentanas e puertas de la [dicha] obra, según está firmada de su nonbre, e de mí, el escribano ynfraescrito, e del dicho Juan Pérez, al qual, el dicho Juan Bautista [dixo] e declaró ante mí, escribano e testigos, el entendimiento y declaraçión de todo lo que se contiene e declara en la dicha su memoria e traça, de que yo, escribano, doy fe.

Y después de ansí entendida e bista la dicha traza e memoria hecha por el dicho Juan Bautista e firmada de su nonbre, según dicho es, el dicho Juan Pérez quedó e se obligó, hará y hedeficará en la dicha obra de las dichas casas que ansí [a de] azer al dicho Juan de Baldés, toda la dicha traça enteramente que el dicho Juan Bautista manda hazer. Y lo hará todo según e [como] en ella se contiene e declara, sin que falte de todo [cosa]//11v alguna de la forma e manera, altos y baxos y ancho e largo, y asientos de bentanas e puertas e sillería $>$ y lo más $<$ quel dicho Juan Bautista manda e a mandado por la dicha su traza firmada de su nonbre, so pena que, no lo haziendo ansy, lo bolberá a deshazer, e lo hará a su costa y debaxo de las más penas, condiçiones e 
posturas contenidas en el dicho contrato que antes déste hiziere, ante el dicho Zerezedo, escribano. E haciéndolo ansí, según dicho es, el dicho señor Juan de Baldés, reg[idor,] dio por ninguna la traza que, para la dicha obra, abía dado el dicho Juan de Zerezedo, maeso, la qual estaba obligado a hazer por el dicho contrato antes déste, el dicho Juan Pérez; e le dio por libre e quito a la dicha traza, solamente de la dicha obligaçión que él tiene hecha. Y quedó de le dar los moldes que ubiere menester para hazer la dicha traça e obra, que ansí nuebamente a de hazer, según lo a mandado el dicho [...]Bautista, e cunplirá con él todo lo demás que por el dicho contrato está obligado, según en él se contiene. El qual y lo en esta escritura contenido, anbas partes, cada una dellas por lo que le toca y es obligado a hazer e conplir, quedaron y se obligaron con sus personas e vienes muebles e rayzes, abidos e por aber, de hazer e que harán e conplirán, según son obligados. E [para] el conplimiento de todo ello, ${ }^{8}$ dieron e otorgaron todo su poder conplido a las justiçias de Su Magestad, destos sus reinos e señoríos, de qualquier fuero e jurisdiçión que sean, ${ }^{9}$ a cuya jurisdiçión se sometieron con las dichas sus personas e bienes, renunçiando como renunçiaron su propio fuero, jurisdiçión e domeçilio, e la ley si conbenerid onium judicium, para que por todo remedio e rigor de derecho les conpelan e apremien a lo ansí conplir, según dicho es y en esta escritura y en el contrato por ellos hecho antes déste se contiene e declara, realmente e con efeto, bien, como si por sentençia definitiva de juez conpetente e por él consentida e pasada en cosa juzgada ansí obiese sido juzgado e sentençiado. Sobre lo qual todo renunçiaron todas e qualesquier leis, fueros e derechos e hordenamientos reales, biexos e nuebos, todos en general e cada uno en espeçial. Espeçialmente renunçiaron la ley e derecho en que diçe que general re- nunçiaçión de ley que honbre haga, que non bala.

Testigos que fueron presentes a lo que dicho es: Fernando de Çifuentes, escribano, e Fernando Garçía de Billar, el biexo, e Fernando de Cangas, vezinos y estantes en esta dicha villa e conçejo. $\mathrm{Y}$ los dichos otorgantes, ambos a dos, a los quales yo, escribano, doy fe conozco, lo firmaron de sus nonbres. Ba testado o deçía, "o" " $\mathrm{p}$ ". Pase por testado. Y entre renglones o diçe "y lo más".

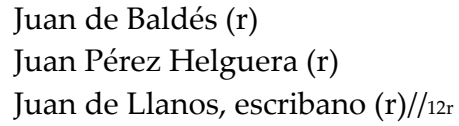

Juan de Baldés (r)

Juan Pérez Helguera (r)

Juan de Llanos, escribano (r)//12r

Condiçiones de la manera que a de hazer Juan Pérez Elguera la obra al señor Juan de Baldés, son las seguientes.

Primeramente asentará la puerta prençipal de las dichas casas más baxo quatro pies quel nibel del corral questá detrás de la dicha ${ }^{10}$ casa; la qual dicha puerta hará conforme al molde e medida questá en el debuxo de esta puerta, y conforme a él, que se entiende que el arco de la dicha puerta de la parte de dentro queda a boluntad del dicho Juan de Baldés sy a de ser de ma ${ }^{11}$ npostería, sy de toba o piedra.

Yten más, será obligado de hazer la puerta del çaguán, para entrar al patio, asentada dos pies más alto que la puerta prençipal. A esta puerta se le dará de ancho çinco pies, e diez de alto, labrada por de dentro e por de fuera, con ${ }^{12}$ el esconze buelto al patio. A de ser quadrada esta puerta, y un pié de grueso los pies derechos y la escarzana de piedra labrada y quadrado.

Yten más, ará dos puertas en el zaguán para que se sirba por ellas las ca-

\footnotetext{
10 Tachado: "s"

11 Tachado: "s"

12 Tachado: "diez los"
}

${ }^{8}$ Tachado: "o"

9 Tachado: "para" 
ballerizas, que serán asentadas al libelo ${ }^{13}$ de la puerta prençipal. Tendrán de ancho quatro pies y medio y de alto, nuebe. Y se entyende que son dos, questán labradas al tienpo que se hazen estas condyçiones.

Yten más ará una puerta que salga a la huerta azia la yglesia, la qual asentara tres pies ${ }^{14}$ más alto que la puerta prençipal, cinco pies de la parte de la quadra que se entyende que en estos çinco pies a de entrar la mitad de la puerta. A de thener de ancho tres pies y medio, y de alto, siete pies, y de esconze y arco de manpostería o toba, a boluntad del dicho Juan de Baldés. Y esta puerta se ará conforme al debuxo e molde que se le da. Y sobre esta puerta hará una saetera a doze pies más alto que la puerta prinçipal.

Yten más, hará seis esquinas en la delantera de las casas [...]quadra, y dos a los rincones conforme $\mathrm{a}[\ldots] / / 12 \mathrm{v}$ rincón, en cada una asentará una saetera, >çinco $<$ seis pies más altas del asiento de la puerta prençipal, e conforme al molde que se le da.

Yten más, ará quatro saeteras, en la delantera ${ }^{15}$ de la casa, doze pies más altos quel asiento de la puerta prençipal, de medio pie de ancho e tres de alto, ras- gada a todas partes, e con desconche ${ }^{16} \mathrm{e}$ arco de manpostería, reserbando que pueda meter en las piedras questán labradas en el esconzie de las otras que están asentadas aora.

Yten más, ${ }^{17}$ desasentará una puerta questá asentada aora en la dicha casa, y la asentará según está allí para salir al corral, asentada al nibelo del corral, en el lugar donde está señalado en la traza de la dicha casa.

Yten más, se a obligado el dicho Juan Pérez Felguera, hazer esta casa conforme a la planta y montea y debuxo que hiço Juan Batysta, que queda en poder del dicho Juan de Baldés, y todas las puertas y bentanas se arán conforme al debuxo ${ }^{18}$ y moldes que se darán al dicho Juan Pérez Felguera. Y entyéndese que todas las escarzanes y esconzes de las dichas bentanas y puertas ${ }^{19} \mathrm{y}$ arcos queda a boluntad de hazerlo el dicho Juan de Helguera, como Juan de Baldés lo quisiere, de manpostería, piedra o toba, o ladryllo.

Ça Batista Portigiani, archizteto (r) Juan Pérez Helguera (r) Juan de Llanos, escribano (r)//
${ }^{13}$ Sic por "nivel"

${ }^{14}$ Tachado: "a"

${ }^{15}$ Corregido sobre "delantedra"
16 Tachado: "de"

17 Tachado: "ará"

18 Tachado: "que"

19 Tachado: "que" 


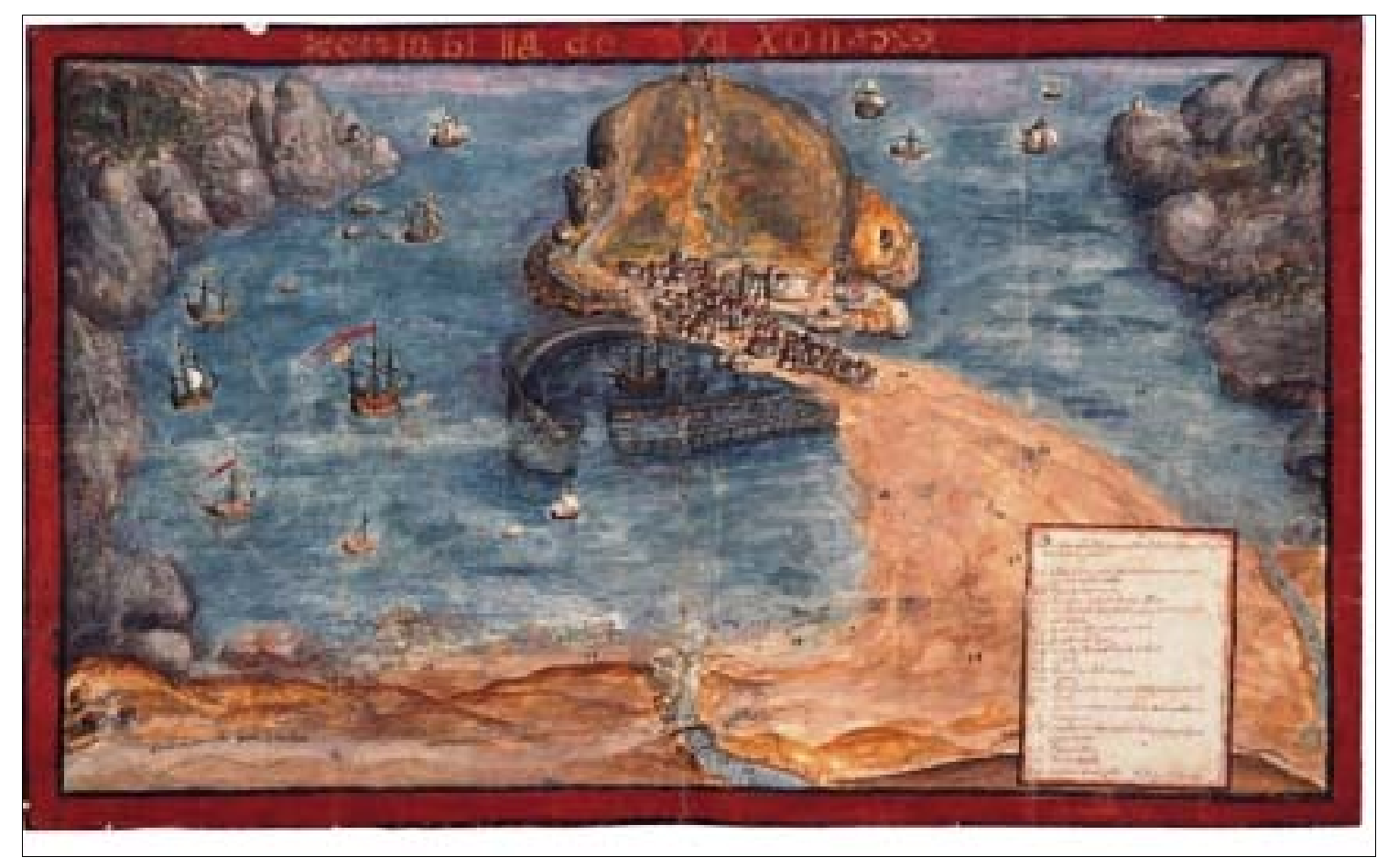

- 1.- (s.f.) [Gijón, 29 de agosto de 1635] Descreçión de la villa de Jijón y del surgidero y las dos puntas que tiene. Tinta y colores a la aguada 507x843 mm. Recuadro de 455x790 mm. AGS.MDP.XXIX-24. Ministerio de Educación Cultura y Deporte. Archivo de Simancas.

"El Grabado de Fernando Valdés, está relacionado con un informe sobre las necesidades de defensa de la villa que este notable Gijónés envió a Felipe IV en 1635"1. En esta imagen se puede apreciar la importancia que tenía ya en esa época el palacio que destaca a la derecha con sus dos torres y su recinto que da a la Iglesia de San Pedro..

${ }^{1}$ F.J. Granda Álvarez, Gijón a Escala. La ciudad a través de su Cartografía, Ayuntamiento de Gijón. Gijón, 2003, pp.16-17. 


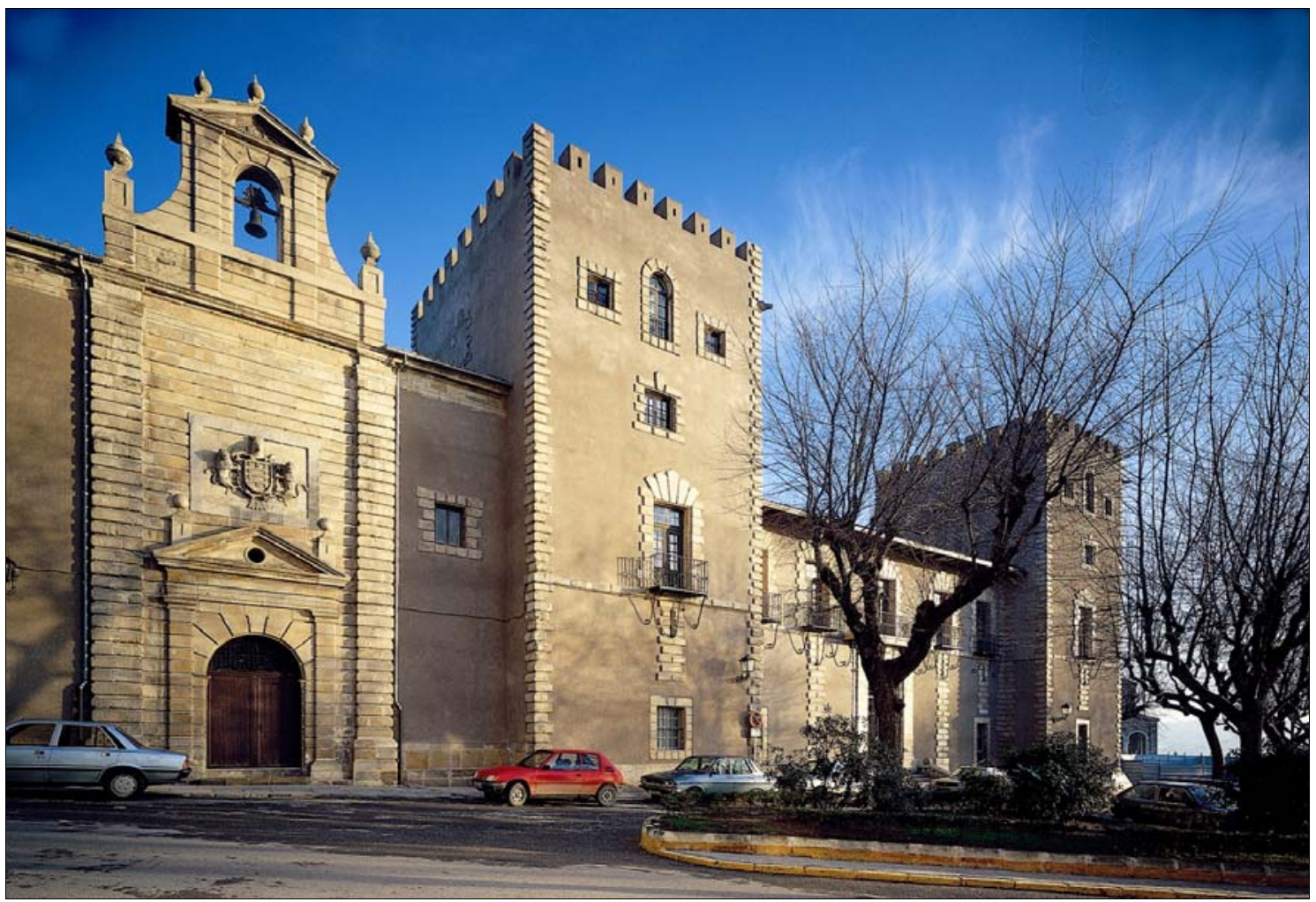

- 2.- Palacio de los Valdés en Gijón. Hoy Colegio Santo Angel. Fotografía publicada en Un paseo con Jovellanos, en el Gijón del siglo XVIII, MâTeresa Caso, Editado por Ayto. de Gijón. Gijón, 2002, P. 16.

Para su descripción y análisis de su arquitectura, acudimos a la ya ofrecida por Germán Ramallo, ${ }^{2}$ sin duda alguna más autorizada y especializada que la yo pueda aportar, y de la que resumo de forma no literal los detalles más esenciales: Un palacio de dos torres con crujía central y capilla, bien separados y definidos buscando claridad estructural. El palacio hoy día está alterado por grandes vanos abiertos en su parte baja. Su cuerpo es de dos pisos, con un tercero, entresuelo, que está oculto pero que es ostensible por las estrechas saeteras bajo los balcones alojadas en las hiladas del almohadillado. Las torres son de cuatro pisos, el último resuelto como variante del "tramo serliano". La única decoración es el almohadillado que recorre en hiladas el piso bajo, las esquinas en todos los volúmenes y enmarca los vanos con variadas soluciones.

\footnotetext{
${ }^{2}$ G. RAMALlO ASENSIO, (Coord). «El palacio urbano en Asturias», Arquitectura señorial en el norte de España, Oviedo, 1993. pp. 86 a 90 .
} 